\title{
Gene Expression in Cumulus Cells and Oocyte Quality
}

\author{
Paolo Giovanni Artini
}

\subsection{Introduction}

Infertility is a worldwide growing issue, and female factors account for about $30 \%$ of the total cases of infertility. The majority of couples affected by infertility undergo in vitro fertilization (IVF) protocols following women hormonal hyperstimulation cycles to obtain mature oocytes to fertilize in vitro. Nowadays, the oocyte/embryo quality is mainly assessed by morphokinetic parameters even if these approaches have low objective prediction value. The rate of live newborns after IVF is relatively low, ranging from about $30 \%$ in younger women to $10 \%$ in the older ones. Aging, in fact, is a well-known critical factor for the success of IVF protocols. As a consequence, a primary goal in older women is to increase the pregnancy rate, with a crucial point represented by the selection of the oocytes to fertilize in vitro and transfer in women.

In this view, transcriptomic information about granulosa cells (GCs) might shed light on the oocyte viability, thereby providing a non-invasive method of oocyte/ embryo selection.

The nutritional support and trafficking of macromolecules that this system allows may be particularly important for oocytes due to the avascular nature of the granulose layer [1]. The signaling between GCs and oocyte via cytoplasmic processes penetrating the zona pellucid and forming gap junctions at the oocyte surface is a key means of disseminating local and endocrine signals to the oocyte [2]. In fact, GCs functionality is a key determinant of the oocyte quality and competence, since GCs are the somatic cells strictly connected to the growing oocyte by a bidirectional communication ensuring the environment for its correct development. It is clear that

P.G. Artini

Division of Gynecology and Obstetrics, Department of Experimental and Clinical Medicine, University of Pisa, Pisa, Italy e-mail: paolo.artini@med.unipi.it 
the role of the oocyte extends far beyond its functions in the transmission of genetic information and supply of raw materials to the early embryo. It also has a critical part to play in mammalian follicular control and the regulation of oogenesis, ovulation rate, and fecundity $[3,4]$.

This chapter is aimed to explore the GCs gene activity in physiological and pathological conditions.

\subsection{Gene Modulation of Granulosa Cells During Folliculogenesis}

The ovarian follicle development is a complex process involving the coordination of many factors that regulate the growth and differentiation of the female gamete and the surrounding somatic components.

Follicular development starts from a pool of inactive primordial follicles. Primordial follicles are generated from primordial germ cells (PGs) and surrounding undifferentiated somatic cells that migrate to the genital ridge where they undergo mitosis cycles creating the germ cell cyst. This process is under the control of many factors such as BMPs, NANOG, OCT4, and FIG $\alpha$.

Following the germ cell cyst, mitosis is arrested and germ cells start meiosis giving rise to the primary oocytes. Primary oocyte and somatic cells form the primordial follicle in which oocytes arrest in the diktyate stage of meiosis I and are surrounded by primordial GCs. This process is regulated by estrogens and a number of growth factors (Ttf and GEFF) and protein (FOXL2 and NOBOX). The activation of primordial follicles to develop in primary follicles is a dynamic process strictly controlled by PI3K/AKT pathway. Primordial follicles are the total germ cells reservoir of a woman and are continuously activated during the entire life to initiate the folliculogenesis. The expression of two oocyte factors (SOHLH1 and NOBOX) is crucial for primordial follicles activation and progression to the primary follicle. During this stage, the oocytes grow and the surrounding GCs begin mitotic divisions. The number of GCs increases as well as the number of cuboidal GCs layers around the oocyte, and the basal lamina expands. GCs express antiMullerian hormone (AMH) to control the number of primordial follicles being active. Primary follicles turn into secondary follicles under the control of local intraovarian factors produced by oocyte and GCs, such as GDF9 and BMP15. The early stages of follicular development are hormones-independent even if GCs express the stimulating hormone receptors (FSHR). Intra-ovarian paracrine factors play their role also during the formation of pre-antral follicle, but the expression of receptors for FSH and LH demonstrates that the follicles become sensitive to gonadotropins at this stage. During the formation of antral follicles, GCs show high proliferative capacity, giving rise to the particular antral multi-layer structure, forming the antral cavity. Many factors are involved in these phases of follicular development including Activin-A and Inhibin $\alpha$. During the antral stage, GCs create the complex network of interaction with oocyte and the other GCs by GAP junctions that are essential for cellular communications during all phases of follicular development. 
Finally, the antral follicle reaches its late stage with the formation of antrum, and GCs differentiate into mural cells (MCs) and cumulus cells (CCs). MCs surround the wall of the follicles and are mainly involved in steroidogenic function, while CCs remain strictly associated to oocyte creating particular gap junctions in a specialized structure, namely, the cumulus-oocyte complex (COC). This particular structure allows the oocyte to acquire the competence to continue meiotic division and the capability to be fertilized. MCs and CCs are differentially regulated by oocyte factors and LH activity, and in particular, oocytes regulate the CCs metabolic activity. Gap junctions are extremely important for the bidirectional communications between oocyte and CCs, which are physically separated by the zona pellucida. These highly specialized junctions allow the passage of many molecules from CCs to oocyte, such as amino acids and metabolites, and their activity is essential to oocyte development and competence. During the ovulation process, CCs expansion is regulated both by oocyte and LH activity [5].

Many pathways have been reported to be activated in the inter-communications between GCs and oocyte, and it is known that alterations in GCs are responsible for oocyte maturation arrest or low quality. The deregulation of GCs translates into follicle microenvironment disruption with oocyte competence and maturation alterations. Furthermore, GCs alterations have been linked to women infertility phenotypes. Transcriptomic analysis of GCs could thus be useful to uncover new biomarkers of oocyte quality and competence [6].

\subsection{Granulosa Cells Transcriptome Analysis}

Given the intimate connection between GCs and oocyte, it is clear that modifications in somatic components transcriptomes affect the oocyte functions and vice versa. The main approaches employed to study GCs transcriptome have been represented by microarray analysis and next-generation RNA sequencing [7]. These two approaches allow to study simultaneously thousands of transcripts and evidence the role of specific genes in normal and pathological oocyte development. Studies performed by using these tools confirmed the role of GCs in steroidogenic function and others processes such as inter-cellular communication and follicle matrix formation, distinguishing the genes selectively expressed by MCs or CCs. Gene expression analyses of GCs in aging women or women with reduced ovarian reserve are of particular interest. They showed the altered expression of key genes involved in glycolytic pathway producing lower levels of progesterone. These conditions could be associated to the inability of GCs to differentiate into mature CCs or to the diminished quality of the oocyte unable to secrete the paracrine factors for GCs differentiation. The increased female age seems to be the most important factor affecting GCs gene expression. Furthermore, GCs transcriptome was reported to be affected by the oocyte aneuploidy, reducing transcriptional activity of the somatic components [8].

GCs gene expression analysis is also useful to differentiate oocyte at a different stage of maturation. The processes mainly modulated by GCs during oocyte 
maturation resulted in the mitogen-activated protein kinase pathway, the lipid biosynsthesis and apoptosis. ANG (angiogenin), PLIN2 (perilipin 2), and RGS2 were indicated as three potential biomarkers of oocyte maturation stage [9].

\subsection{Granulosa Cells Transcriptome Analysis: The Ovarian Stimulation Effect}

Controlled ovarian stimulation (COS) used during assisted reproductive protocols has been indicated as a modifying factor of GCs transcriptome. Many studies reported that ovarian stimulations are able to alter normal CCs gene expression, thus affecting oocyte maturation and competence. In particular, we demonstrated that $\mathrm{r}-\mathrm{hLH}+\mathrm{r}-\mathrm{hFSH}$ and hp-hMG modulate the gene expression of CCs [10]. Probably, the effect of the two preparations at the intracellular level is mediated by different binding affinity of the two molecules with the same receptor, leading in turn to the activation of different pathways. The two treatments differentially alter gene transcription of molecules involved in the trafficking of retinoic acid and ovarian steroidogenesis (RXRB, TTR, ALDH8A1) and follicular development (IL11; AKT3). Few studies analyzed GCs transcriptome modulation by different hormonal stimulations. Recently, Borgbo and colleagues explored the differences in follicle transcriptomes in patients treated with hCG or GnRH by high-density microarray approach. The authors reported that CCs have a higher LHR expression in GnRHatriggered follicles as compared with hCG-triggered follicles while MCs showed the differentially expression of genes like ANGPT1 and SEMA3A, suggesting an impaired induction of angiogenesis [11].

These data demonstrate that GCs, and in particular CCs, analyses could be highly informative about oocyte developmental competence. They could represent a new attractive non-invasive biomarker of oocyte quality. Potential candidate genes, expressed by GCs, which could be used as markers of oocyte quality, are hyaluronan synthase 2 (HAS2), inhibin betaA (INHBA), epidermal growth factor receptor (EGFR), gremlin 1 (GREM1), betacellulin (BTC), CD44, tumor necrosis factorinduced protein 6 (TNFAIP6), and prostaglandin endoperoxide synthase 2 (PTGS2).

\subsection{Granulosa Cells Transcriptome Analysis: Polycystic Ovarian Syndrome}

The data from all of the reviewed investigations showed that the transcriptomic behavior of follicular cells is influenced by a number of variables including body mass index (BMI), serum FSH level and female age, IVF stimulation protocols and disorders. Different studies have been reported a specific gene expression modulation on the follicular cells in patients with polycystic ovarian syndrome (PCOS). PCOS is the most common disorder in women of reproductive age, affecting $7 \%$ of the female population. PCOS shows heterogeneous features characterized by abnormal folliculogenesis. The influence of PCOS on the follicular environment of 
maturing oocytes was assessed by Haouzi et al. [12]. The expression patterns between PCOS patients and control group were significantly different, and multiple genes were affected by PCOS. The members of the growth factor family include EGFR, EREG, and AREG and others known to regulate steroid metabolism such as [AU1] CYP11A1. The authors postulated that the reduced oocyte competence seen in PCOS patients could be due to incorrect functioning of the $\mathrm{Ttf}$ and estrogen receptors signaling cascades.

The central stroma and the granulosa cells were indicated as two possible ovarian sites of the pathophysiological changes in PCOS [13]. It was reported that follicular development disorders in PCOS were mainly related to GCs apoptosis, in association with the increased oxidative stress and reactive oxygen species generation. PCOS transcriptome microarray studies have been performed on whole cultured theca cells, oocytes, and cumulus cells from hyperstimulated and luteinized follicles. Recently, Schmith and colleagues performed an interesting study on ovarian tissue in PCOS patients, where GC and the ovarian central stroma were examined separately. A low-density gene array approach, followed by a real-time PCR validation, was employed to analyze the gene expression in both the central ovarian stroma and in GCs from control and PCOS women. The results showed in the central stroma of PCOS ovaries the down-regulation of five inflammation-related genes (CCL2, IL1R1, IL8, NOS2, TIMP1), the leukocyte marker CD45, the inflammationrelated transcription factor RUNX2 and the growth factor AREG. The growth factor DUSP12 and the coagulation factor TFPI2 were instead over-expressed. On the other hand, PCOS GCs showed the over-regulation of the inflammation-related IL1B, IL8, LIF, NOS2, and PTGS2; the coagulation-related F3 and THBS1; the growth factors BMP6 and DUSP12; the permeability-related AQ3; and the growth arrest-related GADD45A. These data suggested the activation of pro-inflammatory genes in PCOS GCs, which could be validated as markers of follicle maturation defects and predictors of oocyte competence.

\section{Conclusions}

The development of non-invasive oocyte assessments, based on the transcriptomic analysis of GCs, is likely to be of clinical as well as of scientific value. The accurate identification of oocytes that are both chromosomally normal and competent to support early preimplantation development could revolutionize IVF. The data showed suggest that competent oocytes develop in a follicular environment in which processes such as steroidogenesis, cell-cell communication and signaling, metabolism and transport are active. However, this is influenced by a variety of factors. These include the stage of maturation of the developing oocyte, the type of COS employed during IVF and the chromosome constitution of the oocyte.

In conclusion, the improvement of new transcriptomic technologies such as Next Generation Sequencing (RNA-seq) could shed light on the granulosa cells molecular pathways. Nowadays, this technology is not so frequently used compared to microarrays due to its high cost and complex bioinformatic analysis. In any case, these platforms produce a throughput per run that is higher by several 
orders of magnitude versus classical approaches and important discoveries in the field of physiology and/or pathophysiology. These data could be useful to unravel the key genes regulating follicular maturation in physiological and pathological conditions. The use of quantitative gene expression measurements on granulosa cells, which are in close contact with the oocyte during growth and maturation, seems a promising method to predict oocyte quality and competence.

\section{References}

1. Johnson MH (2007) Ovarian function in the adult. In: Johson MH, Everitt BJ (eds) Essential reproduction, 6th edn. Blackwell Scientific Ltd, Oxford, pp 82-91

2. Albertini DF, Combelles CM, Benecchi E, Carabatsos MJ (2001) Cellular basis for paracrine regulation of ovarian follicle development. Reproduction 121:647-653

3. Eppig JJ, Pendola FL, Wigglesworth K, Pendola JK (2005) Mouse oocytes regulate metabolic cooperativity between garnulosa cells and oocytes: amino acid transport. Biol Reprod 73:351-357

4. Gilchrist RB, Lane M, Thompson JG (2008) Oocyte-secreted factors: regulators of cumulus cell function and oocyte quality. Hum Reprod Update 14:159-177

5. Sanchez F, Smitz J (1822) Molecular control of oogenesis. Biochim Biophys Acta 2012: 1896-1912

6. Fragouli E, Lalioti MD, Wells D (2014) The transcriptome of follicular cells: biological insights and clinical implications for the treatment of infertility. Hum Reprod Update 20:1-11

7. Chronowska E (2014) High-throughput analysis of ovarian granulosa cell transcriptome. Biomed Res Int 2014:213570

8. Fragouli E, Wells D, Iager AE, Kayisli UA, Patrizio P (2012) Alteration of gene expression in human cumulus cells as a potential indicator of oocyte aneuploidy. Hum Reprod 27:2559-2568

9. Feuerstein P, Puard V, Chevalier C, Teusan R, Cadoret V, Guerif F, Houlgatte R, Royere D (2012) Genomic assessment of human cumulus cell marker genes as predictors of oocyte developmental competence: impact of various experimental factors. PLoS One 7:e40449

10. Gatta V, Tatone C, Ciriminna R, Vento M, Franchi S, d'Aurora M, Sperduti S, Cela V, Borzì P, Palermo R, Stuppia L, Artini PG (2013) Gene expression profiles of cumulus cells obtained from women treated with recombinant human luteinizing hormone + recombinant human follicle-stimulating hormone or highly purified human menopausal gonadotropin versus recombinant human follicle-stimulating hormone alone. Fertil Steril 99:2000-2008

11. Borgbo T, Povlsen BB, Andersen CY, Borup R, Humaidan P, Grøndahl ML (2013) Comparison of gene expression profiles in granulosa and cumulus cells after ovulation induction with either human chorionic gonadotropin or a gonadotropin-releasing hormone agonist trigger. Fertil Steril 100:994-1001

12. Haouzi D, Assou S, Monzo C, Vincens C, Dechaud H, Hamamah S (2012) Altered gene expression profile in cumulus cells of mature MII oocytes from patients with polycystic ovary syndrome. Hum Reprod 27:89-96

13. Schmidt J, Weijdegård B, Mikkelsen AL, Lindenberg S, Nilsson L, Brännström M (2014) Differential expression of inflammation-related genes in the ovarian stroma and granulosa cells of PCOS women. Mol Hum Reprod 20:49-58 


\section{Author Query}

Chapter No.: 5

0002593039

\begin{tabular}{|c|c|c|}
\hline Query & Details Required & Author's Response \\
\hline AU1 & $\begin{array}{l}\text { Please confirm if changes made to "The members of the } \\
\text { growth factor family include EGFR, EREG and AREG and } \\
\text { others known to regulate steroid metabolism such as } \\
\text { CYP11A1" retained the intended meaning. }\end{array}$ & \multirow{2}{*}{$\begin{array}{l}\text { okay, but in the } \\
\text { line } 155 \text { there is } \\
\text { written "Ttf" but it is } \\
\text { not correct } \\
\text { because it is: } \\
\text { "TGF- } \\
\text { beta" (transforming } \\
\text { growth factor beta) }\end{array}$} \\
\hline & & \\
\hline
\end{tabular}

